\title{
Ferroptotic cell death and TLR4/Trif signaling initiate neutrophil recruitment after heart transplantation
}

\author{
Wenjun Li, ${ }^{1}$ Guoshuai Feng, ${ }^{2}$ Jason M. Gauthier, ${ }^{1}$ Inessa Lokshina, ${ }^{2}$ Ryuji Higashikubo, ${ }^{1}$ Sarah Evans, ${ }^{2}$ Xinping Liu, ${ }^{2}$ Adil Hassan, ${ }^{1}$ \\ Satona Tanaka, ${ }^{1}$ Markus Cicka, ${ }^{2}$ Hsi-Min Hsiao, ${ }^{1}$ Daniel Ruiz-Perez, ${ }^{1}$ Andrea Bredemeyer, ${ }^{2}$ Richard W. Gross, ${ }^{2}$ Douglas L. Mann, ${ }^{2}$ \\ Yulia Y. Tyurina, ${ }^{3}$ Andrew E. Gelman,, ${ }^{1,4}$ Valerian E. Kagan,, ${ }^{3,5}$ Andreas Linkermann, ${ }^{6}$ Kory J. Lavine, ${ }^{2,4,7}$ and Daniel Kreisel ${ }^{1,4}$ \\ 'Department of Surgery and 2Department of Medicine, Washington University, Saint Louis, Missouri, USA. ${ }^{3}$ Department of Environmental and Occupational Health, University of Pittsburgh, Pittsburgh, \\ Pennsylvania, USA. ㄹepartment of Pathology and Immunology, Washington University, Saint Louis, Missouri, USA. ${ }^{5}$ Laboratory of Navigational Redox Lipidomics, I.M. Sechenov Moscow State Medical \\ University, Moscow, Russia. ${ }^{6}$ Department of Internal Medicine III, Division of Nephrology, University Hospital Carl Gustav Carus at Technische Universität Dresden, Dresden, Germany. ${ }^{7}$ Department of \\ Developmental Biology, Washington University, Saint Louis, Missouri, USA.
}

\begin{abstract}
Nonapoptotic forms of cell death can trigger sterile inflammation through the release of damage-associated molecular patterns, which are recognized by innate immune receptors. However, despite years of investigation, the mechanisms that initiate inflammatory responses after heart transplantation remain elusive. Here, we demonstrate that ferrostatin-1 (Fer-1), a specific inhibitor of ferroptosis, decreases the levels of pro-ferroptotic hydroperoxy-arachidonoyl-phosphatidylethanolamine, reduces cardiomyocyte cell death, and blocks neutrophil recruitment following heart transplantation. Inhibition of necroptosis had no effect on neutrophil trafficking in cardiac grafts. We extend these observations to a model of coronary artery ligationinduced myocardial ischemia reperfusion injury (IRI), in which inhibition of ferroptosis resulted in reduced infarct size, improved left ventricular (LV) systolic function, and reduced LV remodeling. Using intravital imaging of cardiac transplants, we show that ferroptosis orchestrates neutrophil recruitment to injured myocardium by promoting adhesion of neutrophils to coronary vascular endothelial cells through a TLR4/Trif/type I IFN signaling pathway. Thus, we have discovered that inflammatory responses after cardiac transplantation are initiated through ferroptotic cell death and TLR4/Trif-dependent signaling in graft endothelial cells. These findings provide a platform for the development of therapeutic strategies for heart transplant recipients and patients who are vulnerable to IRI following restoration of coronary blood flow.
\end{abstract}

\section{Introduction}

Ischemia reperfusion injury (IRI) following heart transplantation is a clinically significant form of sterile inflammation. IRI-mediated primary graft dysfunction complicates the postoperative course of up to $28 \%$ of human cardiac transplant recipients (1). Of note, the development of primary graft dysfunction can result in an early mortality rate of approximately $10 \%$ and is also associated with significantly lower survival rates 1 year after transplantation (2). While several donor and recipient factors have been identified as risk factors for the development of primary graft dysfunction after heart transplantation, the cellular and molecular mechanisms by which IRI induces myocardial inflammation are not well understood.

Neutrophils are recruited to sites of sterile inflammation. For example, our group and others have reported that neutrophils are critical mediators of primary graft dysfunction after lung transplantation (3). Similarly, neutrophils are also recruited to injured

Authorship note: KJL and DK are co-senior authors.

Conflict of interest: DK serves on The Scientific Advisory Board of and receives research support from Compass Therapeutics. DK and KJL have a pending patent entitled "Compositions and methods for detecting CCR2 receptors" (US patent application no. 15/611,577)

Copyright: @ 2019, American Society for Clinical Investigation

Submitted: November 26, 2018; Accepted: February 21, 2019; Published: April 29, 2019

Reference information: J Clin Invest. 2019;129(6):2293-2304

https://doi.org/10.1172/JCl126428. myocardium in humans and experimental animals, in which they cause tissue damage (4). It is the prevailing notion that neutrophil recruitment during sterile injury is triggered through the release of damage-associated molecular patterns (DAMPs) from dead cells and subsequent engagement of innate immune receptors such as TLRs (5). TLR activation initiates signaling via the adaptor proteins MyD88 or Trif. Our current understanding is that, following IRI, cells die through a combination of apoptosis and nonapoptotic forms of programmed cell death (6). It is further postulated that nonapoptotic forms of programmed cell death such as necroptosis or ferroptosis result in the release of DAMPs that ultimately result in the initiation of inflammation, referred to as necroinflammation (7). The precise cell death pathways and signaling events that orchestrate early inflammation after heart transplantation are unknown.

The cellular and molecular requirements that regulate specific steps of neutrophil trafficking differ among various tissues and organs (8). Our laboratory has developed methods to image beating murine hearts by intravital 2-photon microscopy, which allows for a detailed analysis of leukocyte trafficking in cardiac grafts in real time (9). Using this approach, we have recently described that neutrophil extravasation into reperfused transplanted hearts is mediated by tissue-resident CCR2 ${ }^{+}$macrophages through a TLR9/MyD88-dependent pathway (10). Here, we took advantage of intravital imaging to examine the pathways that regulate the 


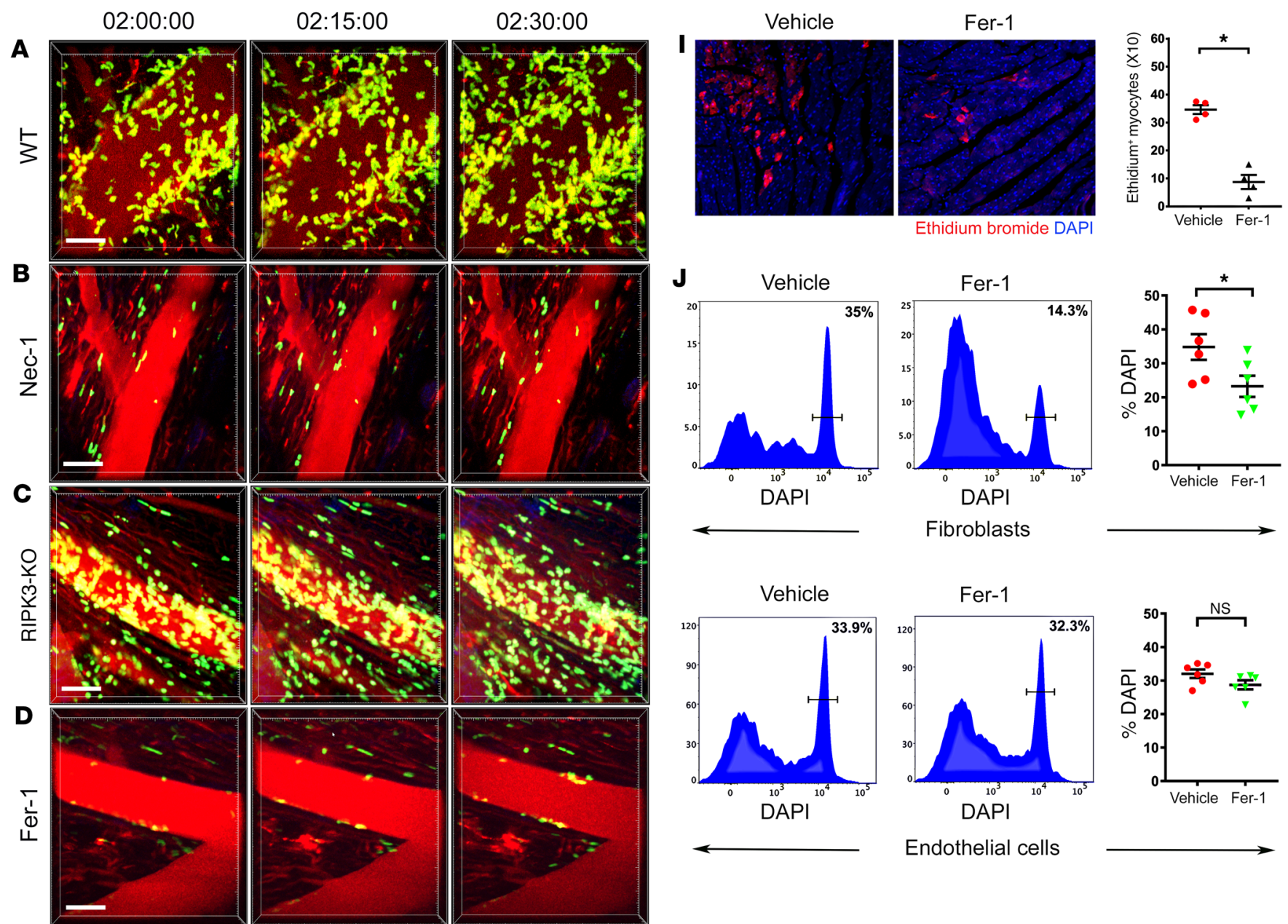

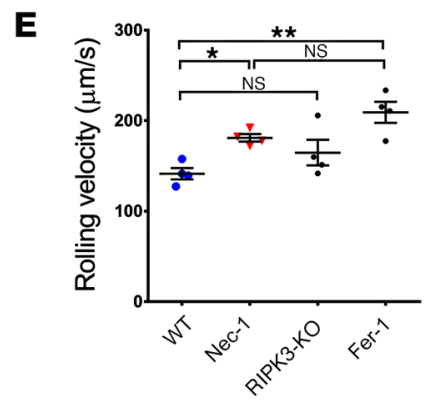

G

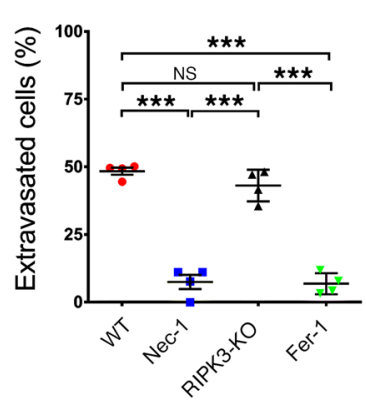

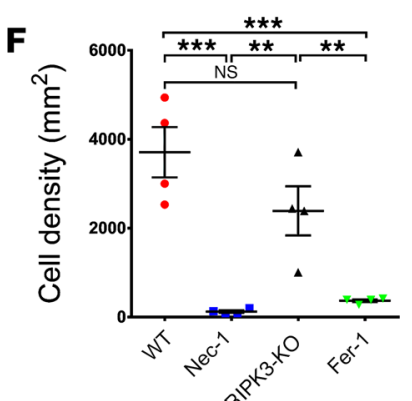

H

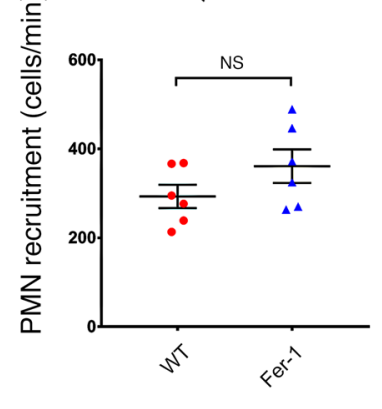

K

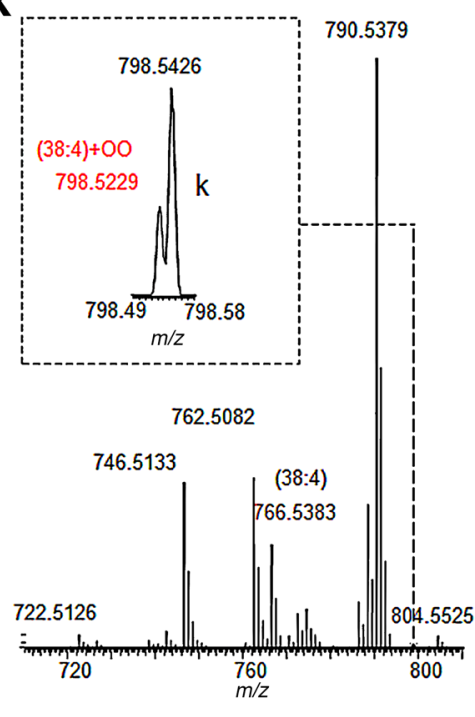

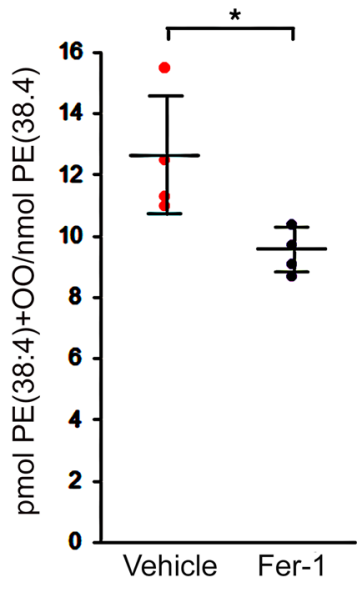


Figure 1. Ferroptosis regulates cell death and promotes neutrophil recruitment in injured hearts. Intravital 2-photon imaging of neutrophil (green) behavior in (A) control WT cardiac grafts, (B) after treatment of recipient mice with Nec-1, (C) in RIPK3-deficient donor hearts, and (D) after administration of Fer-1 to heart recipients. Vessels were labeled red after injection of quantum dots. $n=4$ per experimental group. Scale bars: $30 \mu \mathrm{m}(\mathbf{A}-\mathbf{D})$. (E) Intravascular rolling velocities of neutrophils, (F) density of neutrophils, and (G) percentage of extravasated neutrophils in the experimental conditions displayed in $\mathbf{A}-\mathbf{D}$. Data in E-G represent the mean \pm SEM. ${ }^{*} P<0.05,{ }^{* *} P<0.01$, and ${ }^{* * *} P<0.001$, by 1 -way ANOVA followed by post hoc Tukey's multiple comparisons test. (H) Neutrophil recruitment per minute to coronary veins in control cardiac grafts and after treatment of recipient mice with Fer-1. (I) Cardiomyocyte death determined by ethidium bromide in cardiac grafts after treatment of recipient mice with vehicle or Fer-1. $n=4$ per experimental group. Original magnification, $\times 200$. (J) Flow cytometric assessment of death (DAPI+) of fibroblasts and endothelial cells in B6 cardiac grafts after transplantation into vehicle- or Fer-1-treated syngeneic recipient mice. $n=6$ per experimental group. (K) LC-MS/MS assessment of pro-ferroptotic PE molecular species. MS spectrum of PE obtained from WT mice. Inset: MS spectrum in the range of $\mathrm{m} / \mathrm{z}$ from 798.49 to 798.58 . The content of $\mathrm{PE}(38: 4)+00$ molecular species (HOO-AA-PE, ferroptotic cell death signal) in cardiac grafts after treatment of recipient mice with vehicle or Fer-1. Data in $\mathbf{H}-\mathbf{K}$ represent the mean $\pm \mathrm{SEM} .{ }^{*} P<0.05$, by 2 -sided Mann-Whitney $U$ test.

initial recruitment of neutrophils to cardiac grafts. We discovered that the early cues allowing neutrophils to slow down in the vasculature and to adhere to the vessel wall during IRI are mediated through ferroptotic cell death and TLR4/Trif-dependent signaling in graft endothelial cells.

\section{Results}

Ferroptosis regulates cell death and neutrophil recruitment after heart transplantation. To examine the initial recruitment of neutrophils by intravital 2-photon imaging following cardiac transplantation, we engrafted B6 WT hearts into syngeneic B6 LysM-GFP neutrophil reporter mice $(9,10)$. Consistent with our previous reports, neutrophils were rapidly recruited to heart grafts immediately following reperfusion (Figure 1A and Supplemental Video 1; supplemental material available online with this article; https://doi.org/10.1172/ JCI126428DS1). Only a few cardiomyocytes stained for cleaved caspase-3 in these grafts, and we did not observe differences in neutrophil recruitment kinetics after treating recipient mice with the pan-caspase inhibitor Z-VAD-FMK (Supplemental Figure 1 and Supplemental Video 2). These findings indicate that classical apoptotic pathways of cell death in the cardiac graft do not play an important role in the regulation of early neutrophil recruitment following reperfusion. Treatment of heart transplant recipients with the RIPK1 inhibitor necrostatin-1 (Nec-1) at the time of transplantation, however, resulted in significant increases in rolling velocities. Very few neutrophils slowed down sufficiently to transition to adherence to the walls of the coronary veins (Figure 1, B and E; Supplemental Video 3). Consequently, we observed significant reductions in neutrophil density and also an impairment of their extravasation (Figure 1, F and G). Nec-1 has been frequently used to block necroptosis in various experimental systems (11). To test the role of necroptosis genetically, we next transplanted hearts that lacked expression of RIPK3, a kinase that is essential in triggering necroptosis (12). Transplantation of RIPK3-deficient hearts mirrored the neutrophil behavior observed after engraftment of WT hearts (Figure 1, C and E-G and Supplemental Video 4). As reports have shown that Nec-1 can inhibit both necroptosis and ferroptosis, we next studied the ferroptotic pathway of cell death (13). Treatment of heart recipients with ferrostatin-1 (Fer-1), a selective inhibitor of ferroptosis (14), yielded results similar to those observed after administration of Nec-1 (Figure 1, D-G and Supplemental Video 5). Of note, inhibition of ferroptosis did not affect the rate at which neutrophils were recruited to the graft, and Fer-1 treatment did not directly inhibit neutrophil chemotaxis (Figure $1 \mathrm{H}$ and Supplemental Figure 2). Consistent with published reports of cell death in the setting of myocardial IRI, intravascular injection of ethidium homodimer 2 hours after transplantation revealed an uptake of dye into clusters of cardiomyocytes located within the left ventricular (LV) myocardium (Figure 1I). Administration of Fer-1 to recipients at the time of transplantation resulted in marked reductions in ethidium homodimer in cardiomyocytes compared with that seen in controls. Flow cytometric evaluation revealed that Fer-1 also inhibited the death of fibroblasts, but not endothelial cells, in heart grafts during IRI (Figure 1J and Supplemental Figure 3). In addition, through oxidative lipidomics, we determined that a specific lipid peroxidation product, hydroperoxy-arachidonoyl-phosphatidylethanolamine (HOO-C2O:4/C18:0-PE), which acts as a ferroptotic death signal, was reduced in whole-tissue homogenates obtained from cardiac grafts after treatment with Fer-1 (15) (Figure 1K).

Ferroptosis mediates cell death and tissue injury after nontransplant-related myocardial IRI. To determine whether ferroptosis is responsible for cardiomyocyte cell death following IRI in the absence of exogenous immune cell recruitment, we used Langendorff preparations. We observed that hearts subjected to 1 hour of ischemia had a marked increase in total creatinine kinase (CK) activity released over a 30-minute reperfusion period compared with control hearts that were not subjected to ischemia. Hearts treated with Fer-1 showed reduced levels of released total CK activity compared with vehicle-treated control hearts following IRI (Figure 2A). Serial measurements of released CK activity revealed that Fer-1 decreased CK release after 15 minutes of reperfusion, suggesting that ferroptosis may be selectively affecting cardiomyocyte cell death that occurs as a result of IRI rather than ischemia alone (Figure 2B). Arachidonic acid-containing phospholipids are key mediators of ferroptosis, and oxidized arachidonic acid metabolites are associated with ferroptotic cell death (15). Measurement of arachidonic acid metabolites by liquid chromatography tandem mass spectrometry (LC-MS/MS) revealed an increased abundance of several hydroxyeicosatetraenoic acid (HETE) and epoxyeicosatrienoic acid (EET) species as well as prostaglandin D2 in hearts subjected to IRI. Fer-1 treatment resulted in substantial reductions in the abundance of each of these lipid species. Among oxidized arachidonic acid metabolites increased following IRI, Fer-1 reduced the abundance of several species previously implicated as possible markers of ferroptosis, including 5-HETE, 11-HETE, 12-HETE, and 15-HETE $(13,16)$ (Figure 2C). Fer-1 treatment also reduced the abundance of docosanoids and oxidized linoleic acid species, consistent with the described mechanism of action of Fer-1 as a scavenger of peroxidated lipids (17) (Supplemental Figure 4).

To delineate whether ferroptosis also regulates cardiomyocyte cell death in the context of myocardial infarction, we used an in vivo model of closed-chest myocardial IRI (18). Administration of Fer-1 immediately following 90 minutes of IRI resulted in significant 
A

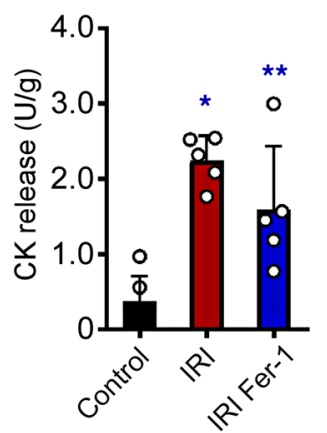

B

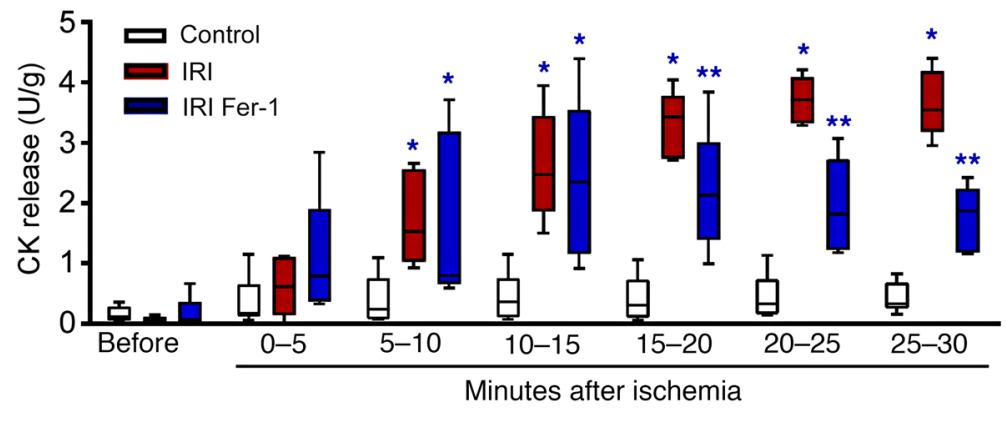

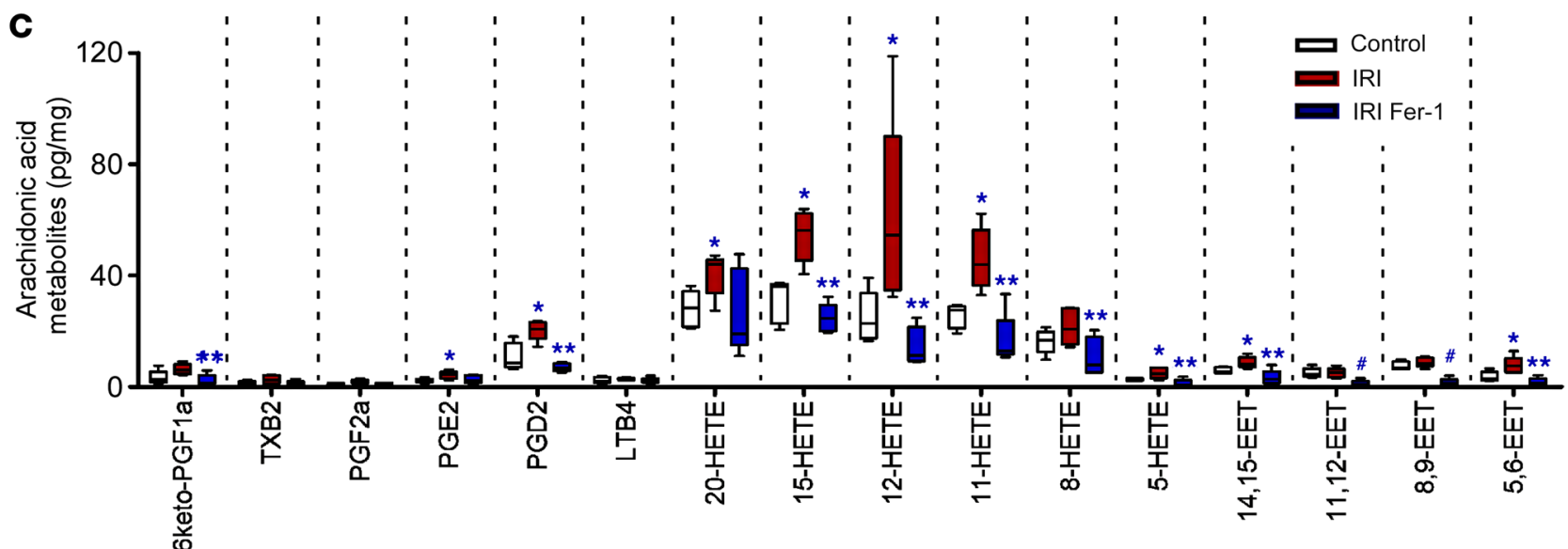

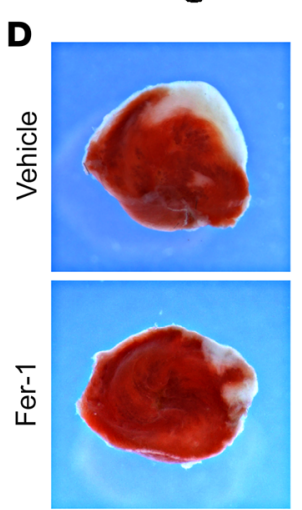

G

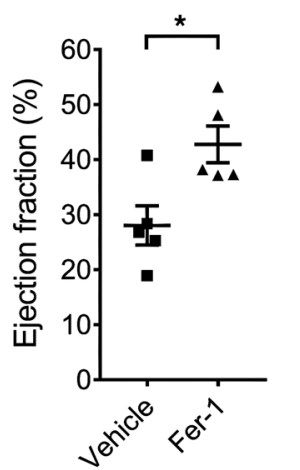

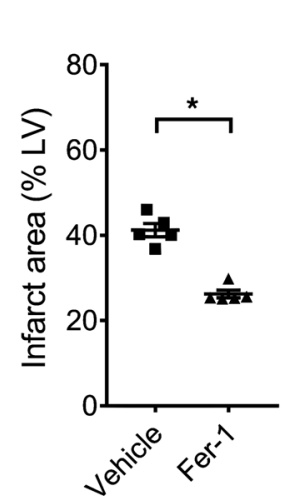

H

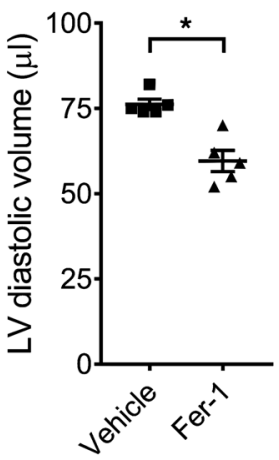

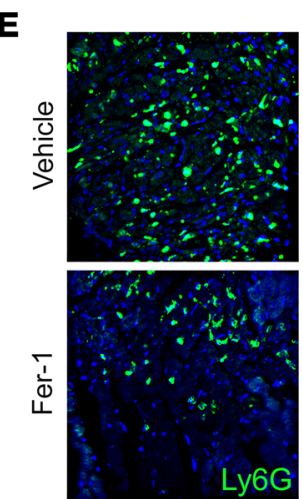

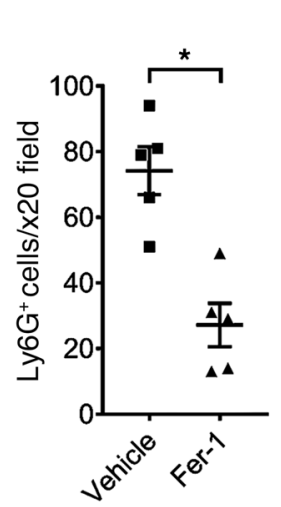

F 4 weeks after MI

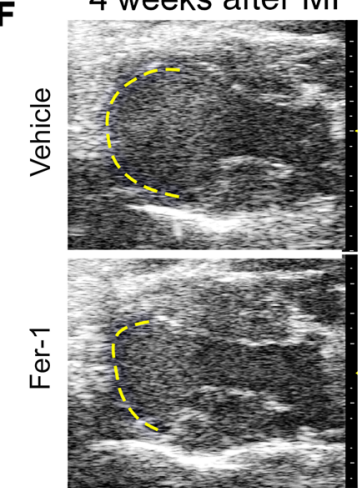

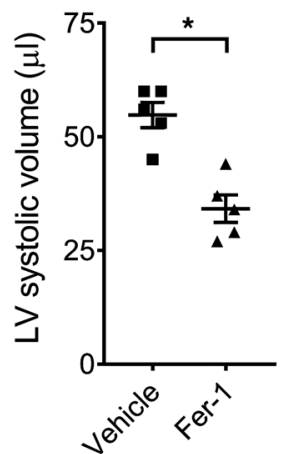

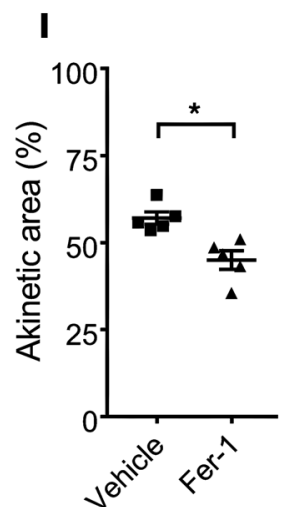

J Vehicle
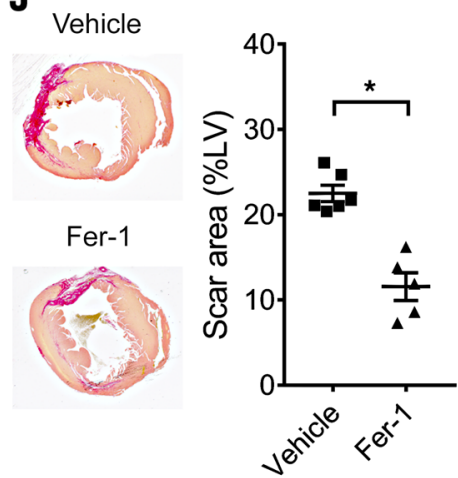
Figure 2. Ferroptosis regulates cardiomyocyte cell death and LV remodeling following myocardial infarction. (A) Measurement of total CK activity in control hearts and hearts subjected to 30 minutes of ischemia followed by 30 minutes of reperfusion using Langendorff preparations. Mice were treated with either vehicle control or Fer- 1 two hours before harvesting. $n=5$ per group. (B) Serial measurement of CK activity following reperfusion in control hearts and vehicle- and Fer-1-treated hearts subjected to IRI. $n=5$ per group. ${ }^{*} P<0.05$ versus control; ${ }^{*} P<0.05$ versus other groups, by 2 -sided Mann-Whitney $U$ test. (C) Arachidonic acid metabolites measured by LC-MS/MS in control hearts and vehicle- and Fer-1-treated hearts subjected to IRI. Data are displayed as box-and-whisker plots. Lines indicate the mean value. ${ }^{*} P<0.05$ versus control; ${ }^{* *} P<0.05$ versus other groups; ${ }^{\#} P<0.05$ versus the vehicle IRI group. (D) TTC staining and measurement of infarct area in hearts obtained from vehicle- and Fer-1-treated mice 48 hours following 90 minutes of IRI. ${ }^{*} P<0.05$ compared with vehicle control. Original magnification, $\times 10$. (E) Ly6G immunostaining and quantification 48 hours following 90 minutes of IRI in vehicle- and Fer-1-treated hearts. Blue indicates DAPI staining. Original magnification, $\times 200$. ${ }^{*} P<0.05$ versus vehicle control. (F) Echocardiography of vehicle- and Fer-1-treated mice 4 weeks following 90 minutes of IRI. Yellow dashed line indicates the akinetic area. (G-I) Quantification of LV ejection fraction, LV diastolic and systolic volumes, and akinetic area in vehicle- and Fer-1-treated mice 4 weeks after IRI. The akinetic area is expressed as a percentage of the LV area. ${ }^{*} P<0.05$ versus vehicle control, by Mann-Whitney $U$ test. (J) Picrosirius red staining (red) and infarct size quantification 4 weeks following 90 minutes of IRI in vehicle- and Fer-1-treated hearts. Lines represent the mean. ${ }^{*} P<0.05$, by 2 -sided Mann-Whitney $U$ test.

reductions in initial infarct area and neutrophil infiltration into the heart 48 hours after myocardial infarction, providing further evidence that ferroptosis is a critical mediator of early cardiomyocyte cell death and neutrophil recruitment in vivo (Figure 2, D and E). Echocardiographic analysis performed 4 weeks after myocardial infarction demonstrated improvements in LV ejection fraction, reductions in LV systolic and diastolic chamber dimensions, and smaller akinetic areas in the hearts of mice treated with Fer-1 (Figure 2, F-I). Pathological analysis 4 weeks after myocardial infarction revealed a smaller infarct size and reduced interstitial fibrosis in Fer-1-treated mice (Figure 2J and Supplemental Figure 5).

Early steps of neutrophil recruitment after heart transplantation are mediated through a TLR4/Trif-dependent pathway. Ferroptosis is an inflammatory form of regulated cell death that results in loss of plasma membrane integrity and the release of DAMPs. Many DAMPs that are released from dying cells in injured tissues elicit responses by signaling through TLR4 (19). Furthermore, TLR4 has been shown to play a role in promoting myocardial IRI (20). Therefore, we next set out to evaluate the role of TLR4 expression in cardiac grafts on neutrophil trafficking in reperfused cardiac grafts. Similar to our observations after treatment of heart recipients with Fer-1 in TLR4-deficient compared with WT hearts, very few neutrophils adhered to the vessel walls, their density and extravasation were significantly reduced, and their rolling velocity was significantly increased (Figure 3, A, B, and E-G, Supplemental Video 5, and Supplemental Video 6). TLR4 can signal via MyD88 or Trif. We have previously shown that neutrophils adhere to the vessel wall but fail to extravasate when heart grafts lack MyD88 expression (10). Thus, MyD88 signaling regulates a step in neutrophil recruitment that is downstream from what we observed in TLR4deficient grafts. Consequently, we investigated the role of Trif signaling in regulating the dynamic behavior of neutrophils in reperfused hearts. Transplantation of hearts that are deficient in CD14, a cell-surface protein that mediates TLR4 internalization and activation of the Trif pathway, or hearts that are deficient in Trif yielded results comparable to those obtained in TLR4-deficient hearts (21) (Figure 3, C-G, Supplemental Video 7, and Supplemental Video 8). To dissect whether TLR4 signaling on hematopoietic or nonhematopoietic graft cells regulates neutrophil recruitment to transplanted hearts, we generated bone marrow chimeras. We found that deletion of TLR4 in hematopoietic graft cells did not have any impact on neutrophil behavior, however, neutrophil adherence to the endothelium and extravasation into the myocardium was significantly impaired when TLR4 was deleted in nonhematopoietic cells (Supplemental Figure 6 and Supplemental Videos 9-11). Given that vascular endothelial cells and cardiomyocytes can signal through TLR4, we next used hearts from TLR4-floxed (TLR $4^{f / f}$ ) donor mice that were crossed with animals in which expression of Cre is driven by an endothelium-specific receptor tyrosine kinase (Tie2) (Tie2-Cre TLR $4^{f / f}$ ) or a cardiac-specific $\alpha$ myosin heavy chain (Myh6) (Myh6-Cre TLR $4^{f / f}$ ) promoter. Elimination of TLR4 on graft vascular endothelial cells, but not on cardiomyocytes, recapitulated the phenotype we have observed in grafts that are globally deficient for TLR4 (Figure 3, H-L, Supplemental Figure 7, and Supplemental Videos 12-14).

Type I IFN signaling regulates the early steps of neutrophil recruitment to transplanted hearts. Trif-mediated signaling stimulates the production of type I IFNs, a process that is regulated by the transcription factors IRF3, IRF5, and IRF7 (22). Donor hearts that lacked expression of type I IFN receptor (IFNAR) or the combination of IRF3, IRF5, and IRF7 displayed increased neutrophil rolling velocity, failure of neutrophils to adhere to the vessel wall, and marked reductions in neutrophil extravasation into the myocardium compared with WT grafts (Figure 4, A-C and F-H, and Supplemental Videos 15 and 16). Neutrophil behavior in these grafts was similar to that seen in Fer-1-treated recipients as well as in TLR4-, CD14-, and Trif-deficient hearts. Consistent with the concept that ferroptosis regulates neutrophil recruitment through a type I IFN-dependent pathway, administration of recombinant IFN- $\alpha$ restored neutrophil recruitment after transplantation into Fer-1-treated hosts (Figure 4, D-H, and Supplemental Video 17).

Neutrophilic infiltration of injured hearts mediates tissue damage. Having shown that ferroptotic cell death regulates the recruitment of neutrophils into injured myocardium, we next set out to examine whether neutrophilic accumulation contributes to cardiac damage. B6 mice that lacked expression of TLR4 on endothelial cells (Tie2-Cre TLR $4^{A / f)}$ ), TLR $4^{f / f l}$ control mice, and neutrophil-depleted B6 mice were subjected to myocardial IRI in an in vivo closed-chest model. We observed significant reductions in neutrophilic infiltration into injured Tie2-Cre TLR $4^{f / / l}$ hearts that were comparable to the levels seen in neutrophil-depleted animals (Figure 5, A and B). We found that either deletion of TLR4 in endothelial cells or depletion of neutrophils resulted in reductions in infarct size 4 days after injury compared with controls (Figure 5, C and D). Echocardiography showed improvements in LV ejection fraction, reductions in LV systolic volume, and smaller akinetic areas in Tie2-Cre TLR $4^{f / l}$ hearts as well as in the hearts of neutrophil-depleted mice compared with controls (Figure 5, E-I). 
A

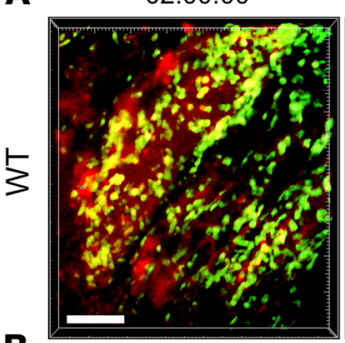

B

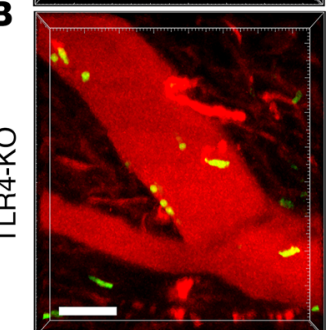

C
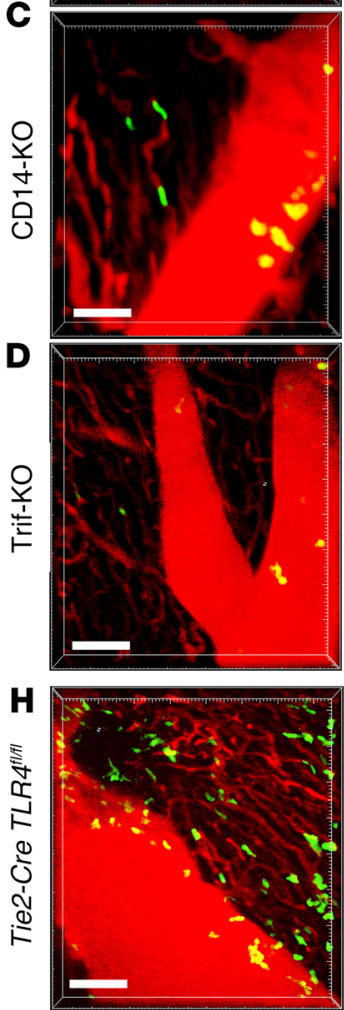

I

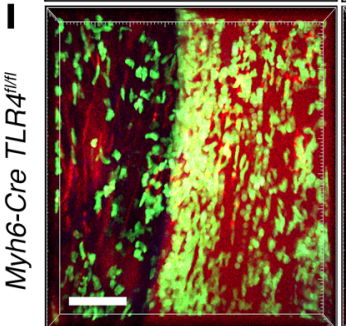

02:15:00
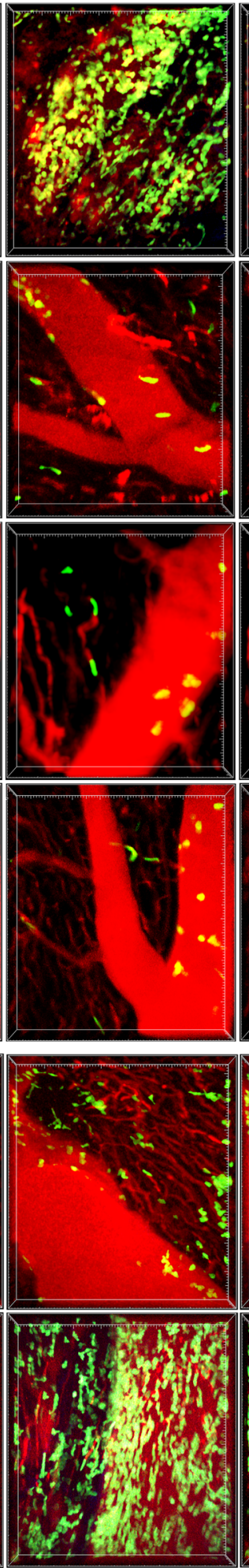

02:30:00
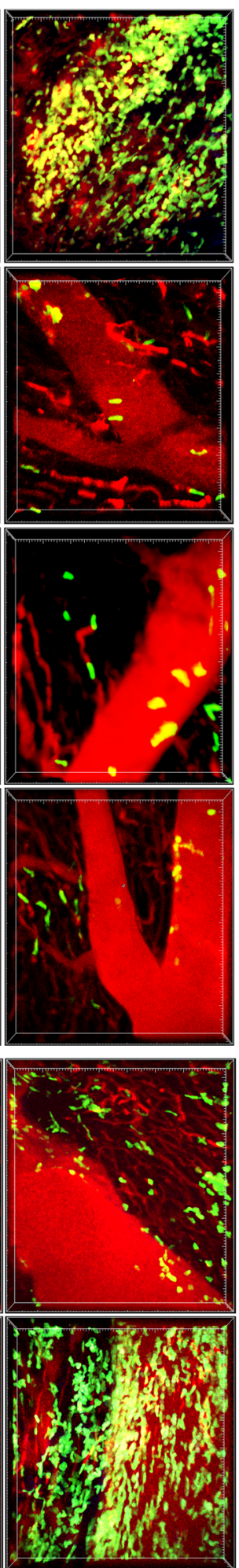

E
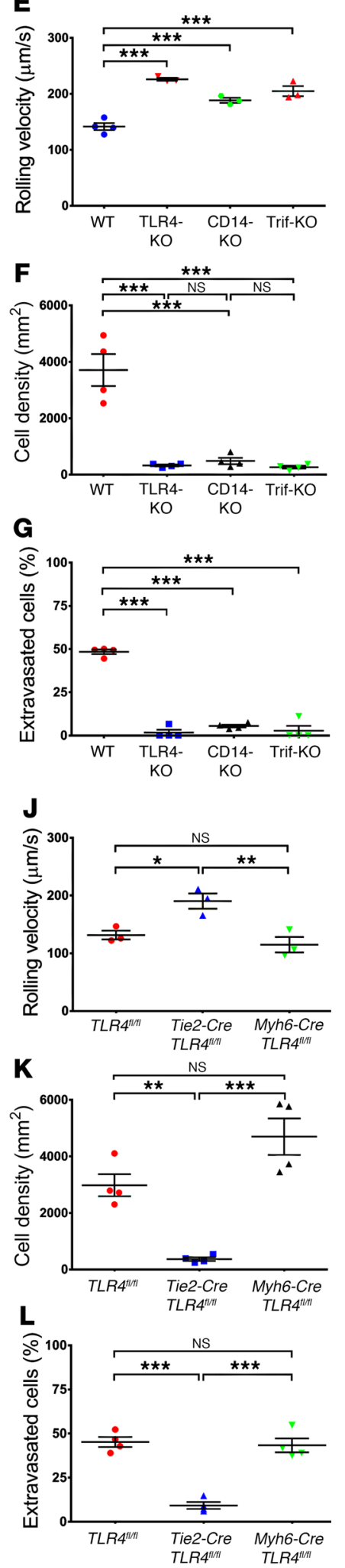

Figure 3. Vascular endothelial TLR4 expression regulates neutrophil recruitment

to injured hearts via Trif signaling. Intravital 2-photon imaging of neutrophil (green) behavior in (A) control WT, (B) TLR4-deficient, (C) CD14deficient, and (D) Trif-deficient cardiac grafts. Vessels were labeled red after injection of quantum dots. $n=4$ per experimental group. (E) Intravascular rolling velocities of neutrophils, (F) density of neutrophils, and (C) percentage of extravasated neutrophils in the experimental conditions displayed in A-D. Intravital 2-photon imaging of neutrophil (green) behavior in hearts that lacked TLR4 expression on $(\mathbf{H})$ vascular endothelial cells (Tie2-Cre TLR $4^{f / / f}$ ) or (I) cardiomyocytes (Myh6-Cre $\left.T L R 4^{f(/ f)}\right)$. (J) Intravascular rolling velocities, (K) density of neutrophils, and (L) percentage of extravasated neutrophils in the experimental conditions displayed in $\mathbf{H}$ and $\mathbf{I}$ as well as control TLR4-floxed heart grafts. Data in E-C and $\mathbf{J}-\mathbf{L}$ represent the mean \pm SEM. ${ }^{*} P<0.05,{ }^{* *} P<0.01$, and ${ }^{* * *} P<0.001$, by 1 -way ANOVA followed by post hoc Tukey's multiple comparisons test. Scale bars: $30 \mu \mathrm{m}$.

\section{Discussion}

Mechanisms guiding leukocyte recruitment differ between various tissues and organs. Intravital 2-photon imaging has enabled us to gain novel insights into cellular and molecular cues that regulate neutrophil recruitment into injured hearts (9). Our observations suggest that endogenous substances released during ferroptotic cell death trigger TLR4 signaling in endothelial cells. More generally, it is likely that ferroptosis results in the release of multiple DAMPs and alarmins that are recognized by a variety of cells within the heart, including immune cells, endothelial cells, and fibroblasts. 
Within endothelial cells, signaling through Trif promotes neutrophil adhesion to coronary veins in a type I IFN-dependent fashion. The failure of neutrophils to adhere to the vessel wall in hearts that do not express TLR4, CD14, Trif, IFNAR, or IRF 3/5/7 resembles our previous observations after inhibition of LFA-1 or CXCL2 $(9,10)$. Engagement of CXCR2 on the surface of neutrophils by CXCL2 presented on the surface of vascular endothelial cells changes the confirmation of the integrin LFA-1, thereby promoting the firm arrest of these neutrophils. Interestingly, type I IFN signaling has been shown to contribute to the production of CXCL2 in models of kidney IRI (23). In contrast to the inability of neutrophils to adhere to the vessel wall when we inhibited ferroptosis or disrupted TLR4/Trif signaling in endothelial cells of injured hearts, we have previously reported that neutrophil recruitment is inhibited further downstream when TLR9/MyD88 signaling is eliminated in tissue-resident cardiac $\mathrm{CCR}^{+}$macrophages. Collectively, these observations indicate that leukocyte trafficking in injured hearts is regulated through various cell populations that signal via different pathways in response to the release of various endogenous ligands.

Our findings extend previous observations regarding the role of TLR4 in promoting IRI in the heart and other organs. Kaczorowski demonstrated that the expression of inflammatory markers was decreased in transplanted hearts after neutralization of HMGB1, an endogenous TLR4 ligand that is released from dying cells, or after elimination of TLR4, CD14, MyD88, or Trif in both the donor and recipient $(24,25)$. Similarly, in a model of myocardial IRI induced by transient coronary artery ligation, cardiac myeloperoxidase (MPO) expression, a surrogate marker for neutrophilic infiltration, is reduced when mice lack expression of TLR4 (26). Using an analogous experimental model, Li showed that hsp60, a DAMP that is released from ischemic myocardium, activates an inflammatory cascade through a TLR4/MyD88-dependent pathway (20). While these studies did not address the question of which cells were activated through TLR4, a few reports have suggested a role for macrophages. Zhu proposed a cascade, whereby DAMPs mediate the production of IL-23 by macrophages through TLR4-dependent signaling in transplanted hearts (27). IL-23 promoted the generation of IL- $17^{+} \gamma \delta$ T cells, which resulted in the recruitment of neutrophils to the graft. TLR4 signaling in hematopoietic cells has also been suggested to mediate hepatic IRI (28). In a model of warm liver IRI, Zhai demonstrated that inflammation was mediated through TLR4 signaling in macrophages, which resulted in MyD88-independent, IRF3-dependent production of type I IFNs $(29,30)$.

The Kubes laboratory has published several reports examining how TLR4 and CD14 expression regulates neutrophil recruitment into the capillary beds of various organs and tissues after local or systemic endotoxin challenges (31-33). Andonegui showed that TLR4 expression by endothelial cells regulates neutrophil sequestration into lung and brain capillaries following systemic administration of LPS $(31,34)$. Tissue-specific variations exist with regard to dependence on CD14 expression for leukocyte sequestration in response to LPS (32). To this end, unlike our observations in reperfused cardiac grafts, endotoxinmediated neutrophil sequestration into the microcirculation of the CNS or liver was not dependent on CD14 expression $(31,33)$.

It has become widely accepted that nonapoptotic forms of cardiomyocyte cell death result in the release of alarmins and DAMPs that ultimately results in the initiation of inflammation, referred to as necroinflammation $(7,35)$. Various cell death signaling pathways have been considered to be involved in myocardial injury. A recent study demonstrated that inhibition of glutaminolysis, a pathway that can induce ferroptotic cell death, resulted in functional improvements in hearts subjected to IRI in an ex vivo preparation (36). Consistent with these observations, we have shown that treatment with Fer-1 reduced the abundance of several oxidized lipids that have been associated with ferroptosis and decreased the release of CK. However, the initial driver of cardiomyocyte death following IRI in vivo has not been conclusively identified. We have demonstrated that ferroptosis mediates early cell death and promotes the initial stages of inflammation following heart transplantation and also during myocardial IRI after transient occlusion of a coronary artery. Intriguingly, we found that cells within the myocardium are differentially responsive to ferroptosis. Cardiomyocytes and fibroblasts, but not endothelial cells, appeared to be most vulnerable to ferroptotic cell death. Ferroptosis has recently been described as a form of programmed cell death that has been implicated in various pathologies including malignancies, neurodegeneration, and IRI involving the kidney and brain (37-41). Biochemically, ferroptosis is dependent on the generation of lipid peroxides, particularly hydroperoxides of polyunsaturated (arachidonoyl) phosphatidylethanolamines (42). Manipulations that interfere with the production of lipid peroxides result in resistance to ferroptosis, and, conversely, inhibition of cellular mechanisms that eliminate lipid peroxides sensitizes cells to ferroptosis $(15,43,44)$. Many of the biochemical mechanisms essential for ferroptosis have been observed in the context of myocardial IRI (45). In addition, enzymatic activities required to execute ferroptosis are also important for cardiomyocyte cell death (46). Notably, a recent study has shown that inhibition of ferroptosis reduces cardiac dysfunction in a model of pressure overload-induced heart failure, suggesting a role for ferroptotic cell death beyond the stages immediately following the injury (47).

Similar to our observations, treatment with Nec-1 has been shown to have a protective effect in models of myocardial IRI (48). However, as Nec-1 can inhibit both necroptosis and ferroptosis, no definitive conclusions about the cell death pathways could be drawn from these investigations. Previous studies using RIPK3deficient mice have suggested that necroptotic cell death may play a role at later time points after heart injury (12). For example, RIPK3-deficient heart allografts showed less cell death, attenuation of lymphocytic infiltrates, and reduced levels of DAMPs 12 days after transplantation into allogeneic hosts (49). We suggest that necroptotic death of graft cells is triggered by tissue-resident or -infiltrating immune cells releasing inflammatory mediators, such as TNF- $\alpha$ (49). This notion is supported by our demonstration that neutrophilic infiltration into injured hearts, a process that is triggered by ferroptosis, augments cell death and mediates cardiac dysfunction. Similarly, following acute kidney injury, necroptosis has been shown to drive a second wave of cell death that amplifies the initial inflammation that is mediated by ferroptosis (50).

In addition to mediating primary graft dysfunction, neutrophils have been shown to enhance alloimmune responses. To this end, it has previously been shown that neutrophilic infiltration into cardiac allografts promotes the subsequent recruitment of alloreactive $\mathrm{T}$ cells (51). Notably, early graft infiltration by memory CD8 ${ }^{+} \mathrm{T}$ cells 


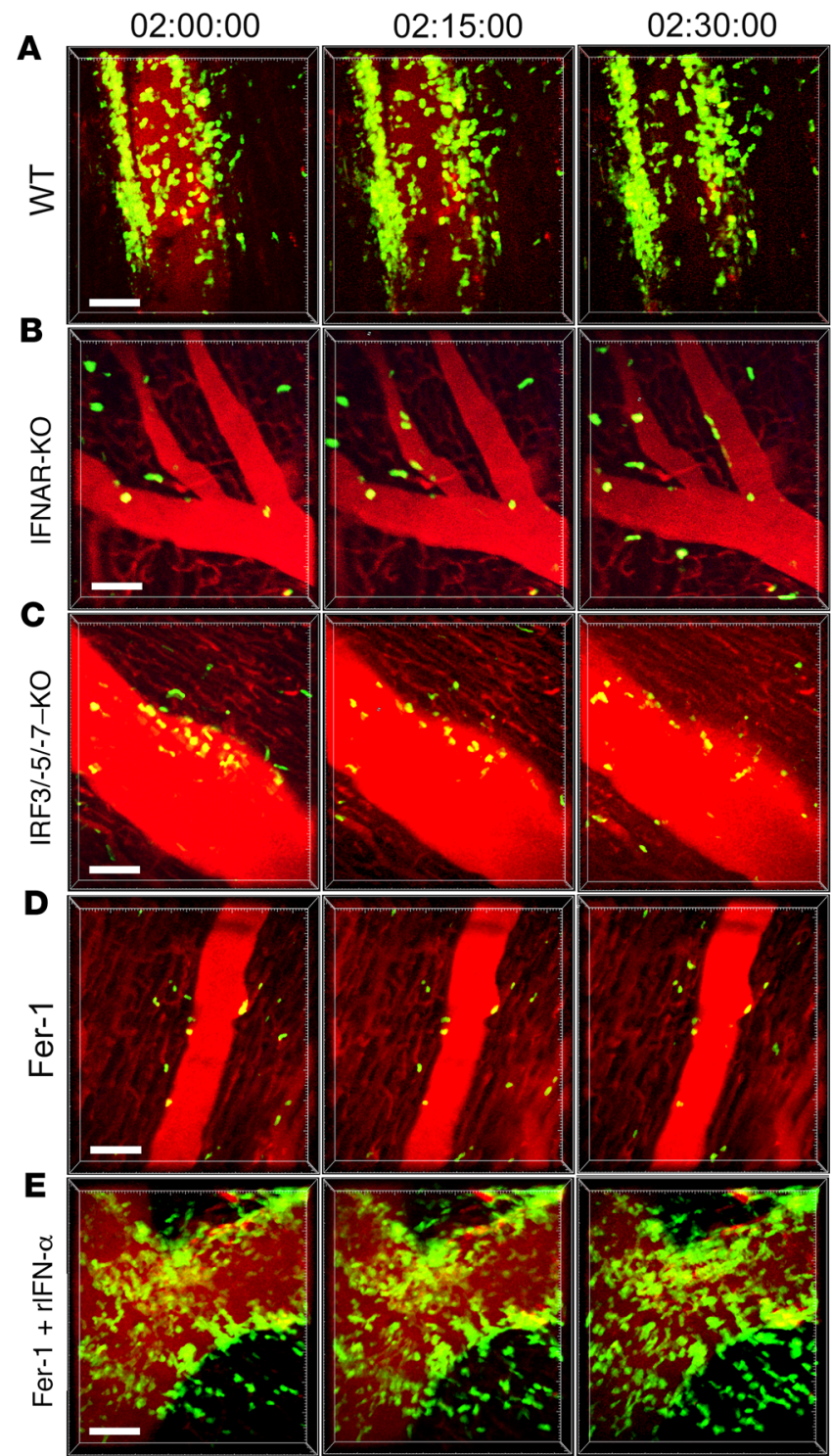

plays an important role in mediating rejection of cardiac allografts that are subjected to prolonged ischemic storage, a process that is known to be associated with enhanced neutrophilic graft infiltration (52). Furthermore, perioperative depletion of neutrophils or inhibition of neutrophil chemotactic pathways synergizes with costimulatory blockade in extending the survival of cardiac allografts (51). Therefore, we speculate that inhibiting ferroptosis in the perioperative period may attenuate alloimmune responses.

In conclusion, our findings provide support for the notion that ferroptotic cell death triggers initial inflammatory responses after heart transplantation. We propose that inhibition of ferroptosis or targeting the TLR4/Trif/type I IFN pathway may be viable clinical strategies to improve outcomes in heart transplant recipients and patients who require reperfusion of ischemic myocardium after coronary occlusion. The recent development of platforms to perfuse and recondition organs ex vivo provides an avenue for the administration of pharmacological agents to donor grafts (53). Our findings suggest that treating donor hearts with a ferroptosis inhibitor prior to transplantation may reduce ischemic injury.

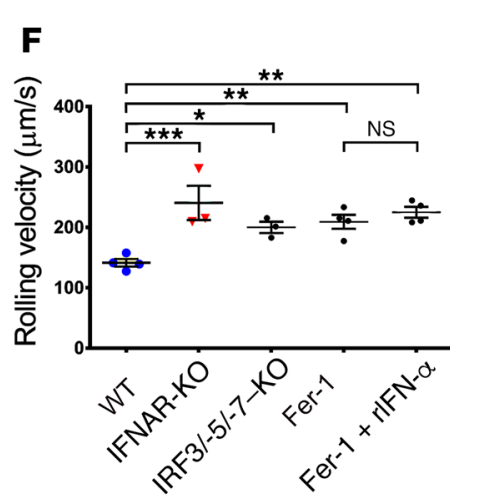

Figure 4. Ferroptosis promotes neutrophil recruitment to injured hearts through type I IFN signaling. Intravital 2-photon imaging of neutrophil (green) behavior in (A) control WT, (B) IFNAR-deficient, and (C) IRF3/-5/-7 triple-deficient heart grafts as well as after transplantation into Fer-1-treated recipients that received (D) vehicle or $(\mathbf{E})$ recombinant type I IFN ( $\mathrm{rIFN}-\alpha)$ at the time of implantation. Vessels were labeled red after injection of quantum dots. $n=4$ per experimental group. (F) Intravascular rolling velocities, (G) density of neutrophils,

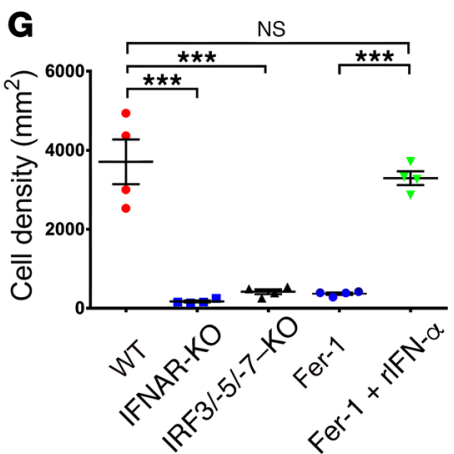
and $\mathbf{( H )}$ percentage of extravasated neutrophils in the experimental conditions displayed in A-E. Data in $\mathbf{F}-\mathbf{H}$ represent the mean $\pm \mathrm{SEM}$. ${ }^{*} P$ $<0.05$, ${ }^{*} P<0.01$, and ${ }^{* *} P<0.001$, by 1-way ANOVA followed by post hoc Tukey's multiple comparisons test. Scale bars: $30 \mu \mathrm{m}$.

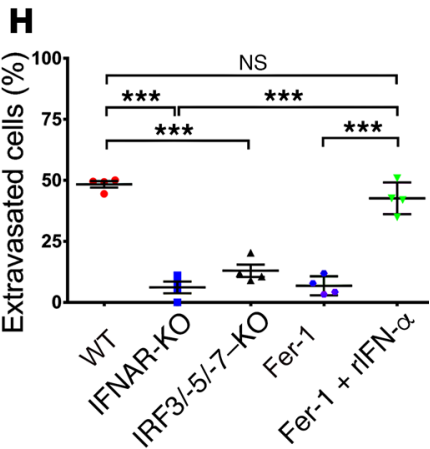

\section{Methods}

Mice. C57BL/6 (B6), B6 TLR4-deficient, B6 CD14-deficient, B6 Trif-deficient, B6 IFNAR-deficient, B6 Tie2-Cre, and B6 Myh6-Cre mice were purchased from The Jackson Laboratory. B6 RIPK3-deficient mice were obtained from Genentech. B6 TLR4-floxed mice were provided by T. Billiar (University of Pittsburgh, Pittsburgh, Pennsylvania, USA), and B6 IRF3/-5/-7 triple-deficient mice were provided by M. Diamond (Washington University, St. Louis, Missouri, USA). B6 LysM-GFP mice were provided by M. Miller (Washington University, St. Louis, Missouri, USA), who had originally obtained them from K. Ley (La Jolla Institute for Allergy and Immunology, La Jolla, California, USA). B6 RIPK3-deficient, B6 TLR4-floxed, and B6 LysM-GFP mice were bred at our facility. B6 TLR4floxed mice were intercrossed with B6 Tie2-Cre or B6 Myh6-Cre mice to generate animals that lacked expression of TLR4 on vascular endothelial cells and cardiomyocytes, respectively. Some recipients were treated with vehicle; Nec-1 (4 mg/kg i.v. 1 hour before and i.p. 2 hours after reperfusion) (MilliporeSigma); Fer-1 (10 mg/kg i.p. 1 hour before reperfusion) (Santa Cruz Biotechnology); Z-VAD-FMK (10 mg/kg i.p. 1 hour before reperfusion) (Santa Cruz Biotechnology); recombinant mouse IFN- $\alpha \mathrm{A}$ 
protein ( $10^{4}$ units i.p. 1 hour before reperfusion) (R\&D Systems); or antimouse Ly6G antibodies (500 $\mu$ g i.p. on day $-1,250 \mu$ i.p. on days 0 and 1) (clone 1A8, Bio X Cell), as indicated for specific experiments. Six- to tenweek-old male and female mice were used for the studies. Sex-matched mice were used for the transplantation procedures.

Heart transplantation. Cardiac grafts were harvested from WT B6 or various gene-deficient donor mice and transplanted into the right neck of B6 LysM-GFP or B6 CD45.1 ${ }^{+}$hosts as previously described (9).

Bone marrow transplantation. Bone marrow-chimeric mice were generated as previously described (54). Briefly, bone marrow was harvested from the femurs of donor mice, and $\mathrm{T}$ lymphocytes were depleted via negative selection with anti-CD90-labeled magnetic microbeads. Recipient mice were lethally irradiated (10 Gy) and subsequently received $1 \times 10^{7} \mathrm{~T}$ cell-depleted donor bone marrow by i.v. injection. Bone marrow chimeras were used as heart donors at least 90 days after the bone marrow transplantation. We have previously reported that this regimen results in near-complete replacement of hematopoietic cells in the heart (54).

IRI. For closed-chest IRI, 2- to 4-month-old B6 mice were anesthetized with sodium pentobarbital, intubated, and mechanically ventilated. The heart was exposed through a midline incision, and an 8-0 prolene suture was placed around the proximal left coronary artery to maximize the ischemic area. The suture was then threaded through a 1-mm piece of polyethylene tubing, forming a loose snare to serve as the arterial occluder. Each end of the suture was exteriorized through the thorax and stored in a subcutaneous pocket. The skin was then closed over the exteriorized suture ends with a 5-0 prolene suture. Instrumented mice were allowed to recover for 2 weeks prior to induction of ischemia. Ischemia was induced after anesthetizing the animals with inhaled $1.5 \%$ isoflurane. The skin was opened over the subcutaneous pocket and the exteriorized suture exposed. The suture ends were dissected away from the subcutaneous tissue, and tension was exerted until ST-segment elevation was seen on the ECG. Ischemia was confirmed by visualizing wall motion abnormalities using simultaneous echocardiography. Following 90 minutes of ischemia, tension was released, and the suture ends were placed back into the subcutaneous pocket. The skin was then closed. Sham animals underwent an identical procedure with the exception that tension was not placed on the suture ends. Vehicle or Fer-1 (0.25 mg i.p.) was administered 24 and 2 hours before IRI.

Echocardiography. The mice underwent echocardiography in the Washington University Mouse Cardiovascular Phenotyping Core facility using the VisualSonics 770 Echocardiography System. 2D and $\mathrm{M}$-mode images were obtained in the long- and short-axis views. Ejection fraction and LV dimensions were calculated using edge-detection software and standard techniques. The akinetic region was calculated by measuring the area of the akinetic portion of the $\mathrm{LV}$ myocardium and normalizing it to the area of total LV myocardium. Measurements were performed on 3 independently acquired images per animal by investigators who were blinded to the experimental groups.

Triphenyltetrazolium chloride staining. Hearts were harvested, perfused with $20 \mathrm{ml}$ ice-cold PBS, and sliced into 4 pieces using a razor blade. Myocardial slices were then immersed in 1\% triphenyltetrazolium chloride (TTC) (MilliporeSigma) diluted in $0.9 \%$ sodium chloride and incubated for 30 minutes on an agitator at $37^{\circ} \mathrm{C}$. Slices were then fixed in $10 \%$ formalin and imaged on a Zeiss stereomicroscope. Quantification of the infarct area was performed using ImageJ software (NIH) in a blinded fashion.
Immunostaining and Picrosirius red staining. For histological analyses, tissues were fixed in $2 \%$ paraformaldehyde (PFA) overnight at $4^{\circ} \mathrm{C}$, dehydrated in $70 \%$ ethyl alcohol, and embedded in paraffin. Sections (4- $\mu \mathrm{m}$ thick) were cut and stained with Picrosirius red using standard techniques. Picrosirius red staining was quantified using ImageJ software. For all immunostaining assays, tissues were fixed in 2\% PFA overnight at $4^{\circ} \mathrm{C}$, embedded in OCT, infiltrated with $30 \%$ sucrose, frozen, and $12-\mu \mathrm{m}$ cryosections cut. The following primary antibodies were used: Ly6G (clone 1A8) 1:100 (BD Biosciences) and cleaved caspase-3 (clone 5A1E) 1:1000 (Cell Signaling Technology). Immunofluorescence was visualized using appropriate secondary antibodies on a Zeiss confocal microscopy system. For all experiments, at least 4 sections from 4 independent samples were analyzed in a blinded fashion.

Flow cytometric analysis. Heart tissue was prepared for flow cytometry as previously described (10). Cells were stained with fluorochrome-labeled anti-CD45 (clone 30-F11, eBioscience); anti-CD31 (clone MEC13.3, BD Biosciences); anti-feeder cells (clone mEF-SK4, Miltenyi Biotec) (55); DAPI (Thermo Fisher Scientific); and isotype control antibodies.

Neutrophil chemotaxis. Neutrophils were isolated from the bone marrow of B6 mice by negative selection as previously described (10). Neutrophils ( $10^{6}$ neutrophils), suspended in $100 \mu \mathrm{l}$ complete RPMI medium containing $10 \%$ FBS, were placed in the upper chamber of a Transwell (6.5 mm, 3- $\mu \mathrm{m}$ pore size, polycarbonate membrane, Thermo Fisher Scientific). Recombinant CXCL2 (c452-M2-010; 100 ng/ml; R\&D Systems) and/or Fer-1 (100 $\mu \mathrm{M})$ were added to complete RPMI medium in the lower chamber. After a 2-hour incubation period at $37^{\circ} \mathrm{C}$ in $5 \% \mathrm{CO}_{2}$, cells collected from the bottom chamber were counted in 10 $\mu \mathrm{l}$ complete RPMI medium using a hemocytometer.

Intravital 2-photon microscopy. Intravital imaging of cardiac grafts was performed using a custom-built 2-photon microscope running ImageWarp version 2.1 acquisition software (A\&B Software) as previously described (9). For time-lapse imaging of neutrophil trafficking in cardiac grafts, we averaged 15 video-rate frames ( 0.5 seconds per slice) during the acquisition to match the ventilator rate and minimize movement artifacts. Each plane represents an image of $220 \times 240 \mu \mathrm{m}$ in the $x$ and $y$ dimensions. Twenty-one sequential planes were acquired in the $z$ dimension (2.5- $\mu$ m each) to form a $Z$-stack. Each individual neutrophil was tracked from its first appearance in the imaging window and followed up to the time point at which it dislocated more than 20 $\mu \mathrm{m}$ from its starting position. To visualize coronary vessels and determine whether neutrophils were intravascular or extravascular, we i.v. injected $50 \mu \mathrm{l}$ PBS containing $15 \mu \mathrm{l} 655$-nm nontargeted Q-dots (Thermo Fisher Scientific) immediately before imaging. To determine the percentage of extravasated neutrophils, the number of extravascular neutrophils was divided by the sum of intravascular and extravascular neutrophils. Multidimensional rendering and manual cell tracking were done with Imaris (Bitplane). Data were transferred and plotted in GraphPad Prism 6.0 for creation of the graphs.

MS analyses of phosphatidylethanolamine and its oxidation products. Lipids were extracted using the Folch procedure (56), and phosphatidylethanolamines (phospholipids) were analyzed by LC-MS/MS (15) using normal phase [Luna $3 \mu \mathrm{m}$ Silica (2), $100 \AA$, $150 \times 2.0 \mathrm{~mm}$; Phenomenex] at a flow rate of $0.2 \mathrm{ml} /$ minute on a Dionex Ultimate 3000 HPLC System (Thermo Fisher Scientific) at $35^{\circ} \mathrm{C}$. Gradient solvents A: propanol/hexane/water (285:215:5, v/v/v) and B: propanol/hexane/ water (285:215:40, v/v/v) containing $10 \mathrm{mM}$ ammonium acetate were 
A
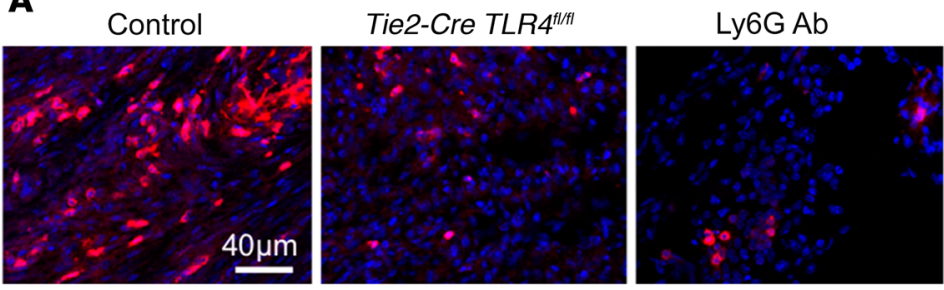

Ly6G DAPI
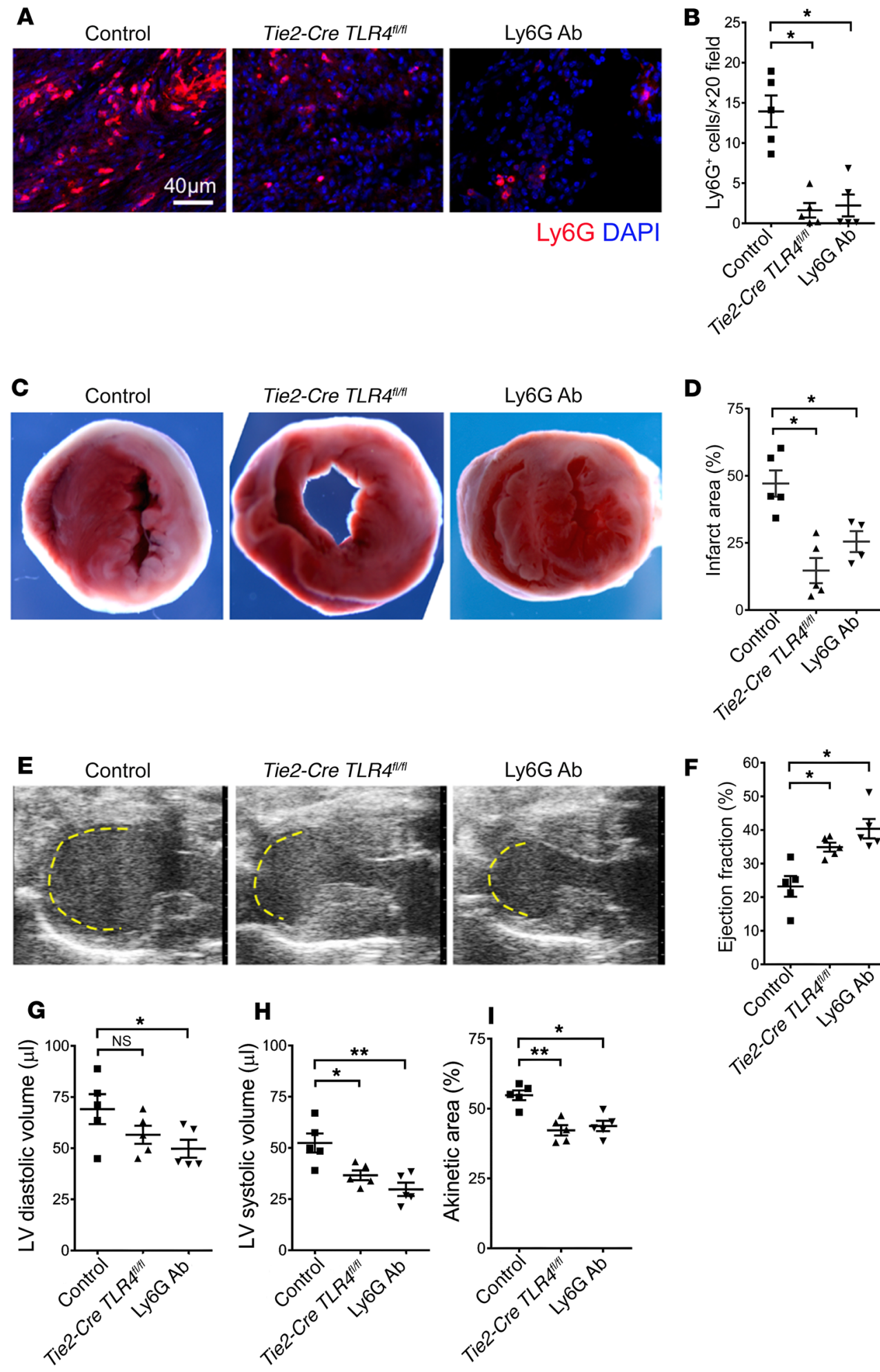

D

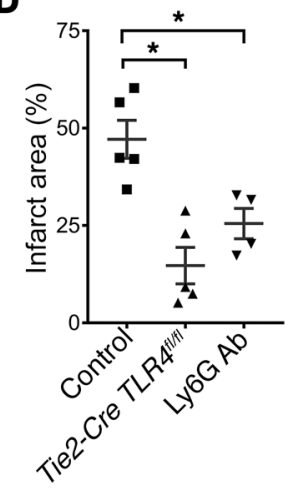

F

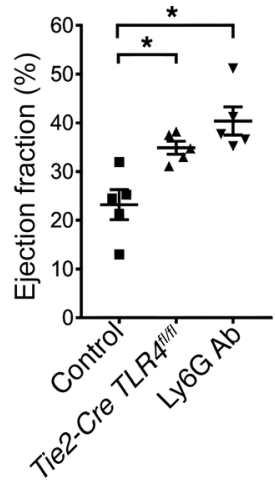

Figure 5. Neutrophilic infiltration contributes to cardiac damage. (A) Ly6C immunostaining and (B) quantification 4 days following 90 minutes of closed-

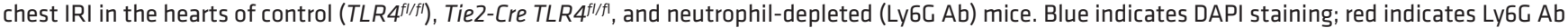

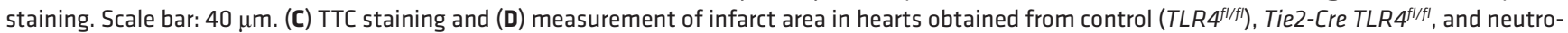
phil-depleted (Ly6C Ab) mice 4 days following 90 minutes of closed-chest IRI. Original magnification, $\times 10$. (E) Echocardiography of control (TLR4 fl/ff), TiezCre TLR4 fl/fl, and neutrophil-depleted (Ly6C Ab) mice 4 days following 90 minutes of IRI. Yellow dashed line indicates the akinetic area. (F-I) Quantification of LV ejection fraction, LV diastolic and systolic volumes, and akinetic area in control (TLR4 f//ff), Tiez-Cre TLR $4^{f / / f l}$, and neutrophil-depleted (Ly6C Ab) mice 4 days following 90 minutes of IRI. The akinetic area is expressed as a percentage of the LV area. $n=4-5$ per experimental group. Lines represent the mean. ${ }^{*} P<0.05$ and ${ }^{* *} P<0.01$, by 1-way ANOVA followed by post hoc Tukey's multiple comparisons test. 
used. The column was eluted for 0 to 23 minutes with a linear gradient from $10 \%$ to $32 \% \mathrm{~B} ; 23-32$ minutes from $32 \%$ to $65 \% \mathrm{~B} ; 32-35$ minutes from $65 \%$ to $100 \% \mathrm{~B} ; 35-62$ minutes held at $100 \% \mathrm{~B} ; 62-64$ minutes from $100 \%$ to $10 \% \mathrm{~B}$ followed by equilibration from 64 to 80 minutes at $10 \%$ B. MS and $\mathrm{MS}^{2}$ analysis was performed on a Q-Exactive hybrid quadrupole orbitrap mass spectrometer (Thermo Fisher Scientific) in negative ion mode at a resolution of 140,000 for the full MS scan and 17,500 for the $\mathrm{MS}^{2}$ scan in a data-dependent mode. Analysis of data was performed using the software package Compound Discoverer (Thermo Fisher Scientific) with an in-house-generated analysis workflow and oxidized phospholipid database. Phospholipids were filtered by retention time and confirmed by fragmentation analysis.

MS analyses of eicosanoids, docosanoids, and oxidized metabolites of linoleic acid. MS analyses of signaling eicosanoids, docosanoids, and oxlams were performed using a charge-switch strategy by derivatization with $\mathrm{N}$-(4-aminomethylphenyl) pyridinium (AMPP) and subsequent LC-MS/ MS with multiple reaction monitoring (MRM) and accurate mass determination of diagnostic product ions as previously described (57).

IRI ex vivo (Langendorff preparations). Hearts from vehicle control and Fer-1-treated (0.25 mg i.p. injected 24 and 2 hours before harvest) B6 mice were isolated and perfused at a constant pressure of $70 \mathrm{mmHg}$ with modified Krebs-Henseleit buffer. After a 20-minute stabilization period, hearts were subjected to no-flow ischemia ( $t=0$ minutes) for 30 minutes followed by reperfusion ( $t=30$ minutes) for up to $60 \mathrm{~min}$ utes ( $t=90$ minutes). To assess the degree of cardiomyocyte injury after IRI ex vivo, we measured CK release in the effluent collected every 5 minutes during reperfusion using a commercially available CK assay kit (catalog 326-10; Sekisui Diagnostics) according to the manufacturer's recommendations. CK activity was normalized for dry heart weight, and data are expressed as units per gram of cardiac tissue.

Statistics. Differences between groups were compared using a 2-sided Mann-Whitney $U$ test. Multiple means were compared using a 1-way ANOVA followed by post hoc Tukey's multiple comparisons test. A $P$ value of less than 0.05 was indicative of a statistically significant difference. Data were analyzed using GraphPad Prism, version 6.0-7.0 (GraphPad Software) and are presented as dot plots or boxand-whisker plots generated in GraphPad Prism. Statistical tests and exact sample sizes used to calculate statistical significance are stated in the figure legends.
Study approval. Animal experiments were approved by the Institutional Animal Studies Committee at Washington University. Animals received humane care in compliance with the Guide for the Care and Use of Laboratory Animals prepared by the National Academy of Sciences (National Academies Press, 2011) and published by the NIH and with the Principles of Laboratory Animal Care formulated by the National Society for Medical Research.

\section{Author contributions}

WL performed the heart transplantation and intravital 2-photon imaging studies; JMG, AH, and ST performed analysis of intravital 2-photon imaging studies; RH and DRP performed flow cytometric analyses; $\mathrm{HMH}$ performed chemotactic assays; $\mathrm{GF}$ and $\mathrm{AB}$ performed experiments in the myocardial infarction model; SE, IL, and MC performed experiments involving Langendorff preparations; XL and RWG performed MS analyses of eicosanoids, docosanoids, and oxidized metabolites of linoleic acid; YYT and VEK performed MS analyses of phosphatidylethanolamine and its oxidation products; DLM, AL, and AEG assisted with experimental design and critical review of the manuscript; KJL and DK are responsible for all aspects of this work including experimental design, data analysis, and manuscript production.

\section{Acknowledgments}

DK is supported by the NIH (P01AI116501 and R01HL094601), a Veterans Administration Merit Review grant (1I01BX002730), and the Foundation for Barnes-Jewish Hospital. KJL is supported by the NIH (R01 HL138466, R01 HL139714, and K08 HL123519) and the Burroughs Wellcome Fund (grant 1014782). Histology was performed in the DDRCC advanced imaging and tissue analysis core, which is supported by NIH grant P30 DK52574.

Address correspondence to: Daniel Kreisel, Department of Surgery, 660 South Euclid, Campus Box 8234, St. Louis, Missouri 63110, USA. Phone: 314.362.6021; Email: kreiseld@wustl.edu. Or to: Kory J. Lavine, Division of Cardiology, 660 South Euclid, Campus Box 8086, St. Louis, Missouri 63110, USA. Phone: 314.362.1171; Email:klavine@wustl.edu.
1. Kobashigawa J, et al. Report from a consensus conference on primary graft dysfunction after cardiac transplantation. J Heart Lung Transplant. 2014;33(4):327-340.

2. Nicoara A, et al. Primary graft dysfunction after heart transplantation: Incidence, trends, and associated risk factors. Am J Transplant. 2018;18(6):1461-1470.

3. Kreisel D, et al. Bcl3 prevents acute inflammatory lung injury in mice by restraining emergency granulopoiesis. JClin Invest. 2011;121(1):265-276.

4. Ma XL, Tsao PS, Lefer AM. Antibody to CD-18 exerts endothelial and cardiac protective effects in myocardial ischemia and reperfusion. J Clin Invest. 1991;88(4):1237-1243.

5. McDonald B, et al. Intravascular danger signals guide neutrophils to sites of sterile inflammation. Science. 2010;330(6002):362-366.

6. Wu MY, et al. Current mechanistic concepts in ischemia and reperfusion injury. Cell Physiol Biochem. 2018;46(4):1650-1667.

7. Sarhan M, Land WG, Tonnus W, Hugo CP, Linkermann A. Origin and Consequences of Necroinflammation. Physiol Rev. 2018;98(2):727-780.

8. Kolaczkowska E, Kubes P. Neutrophil recruitment and function in health and inflammation. Nat Rev Immunol. 2013;13(3):159-175.

9. Li W, et al. Intravital 2-photon imaging of leukocyte trafficking in beating heart. J Clin Invest. 2012;122(7):2499-2508.

10. Li W, et al. Heart-resident CCR2. JCI Insight. 2016;1(12):87315.

11. Kanou T, et al. Inhibition of regulated necrosis attenuates receptor-interacting protein kinase 1-mediated ischemia-reperfusion injury after lung transplantation. J Heart Lung Transplant. 2018;37(10):1261-1270.

12. Luedde M, et al. RIP3, a kinase promoting necro- ptotic cell death, mediates adverse remodelling after myocardial infarction. Cardiovasc Res. 2014;103(2):206-216.

13. Friedmann Angeli JP, et al. Inactivation of the ferroptosis regulator $\mathrm{Gpx} 4$ triggers acute renal failure in mice. Nat Cell Biol. 2014;16(12):1180-1191.

14. Zilka O, et al. On the Mechanism of cytoprotection by ferrostatin-1 and liproxstatin- 1 and the role of lipid peroxidation in ferroptotic cell death. ACS Cent Sci. 2017;3(3):232-243.

15. Kagan VE, et al. Oxidized arachidonic and adrenic PEs navigate cells to ferroptosis. Nat Chem Biol. 2017;13(1):81-90.

16. Gaschler MM, Stockwell BR. Lipid peroxidation in cell death. Biochem Biophys Res Commun. 2017;482(3):419-425.

17. Skouta R, et al. Ferrostatins inhibit oxidative lipid damage and cell death in diverse disease models. J Am Chem Soc. 2014;136(12):4551-4556. 
18. Dewald O, et al. Downregulation of peroxisome proliferator-activated receptor-alpha gene expression in a mouse model of ischemic cardiomyopathy is dependent on reactive oxygen species and prevents lipotoxicity. Circulation. 2005;112(3):407-415.

19. Land WG, Agostinis P, Gasser S, Garg AD, Linkermann A. Transplantation and Damage-Associated Molecular Patterns (DAMPs). Am J Transplant. 2016;16(12):3338-3361.

20. Li Y, et al. Myocardial ischemia activates an injurious innate immune signaling via cardiac heat shock protein 60 and Toll-like receptor 4. J Biol Chem. 2011;286(36):31308-31319.

21. Zanoni I, et al. CD14 controls the LPS-induced endocytosis of Toll-like receptor 4. Cell. 2011;147(4):868-880.

22. Lazear HM, et al. IRF-3, IRF-5, and IRF-7 coordinately regulate the type I IFN response in myeloid dendritic cells downstream of MAVS signaling. PLoS Pathog. 2013;9(1):e1003118.

23. Freitas MC, Uchida Y, Lassman C, Danovitch GM, Busuttil RW, Kupiec-Weglinski JW. Type I interferon pathway mediates renal ischemia/reperfusion injury. Transplantation. 2011;92(2):131-138.

24. Kaczorowski DJ, et al. Toll-like receptor 4 mediates the early inflammatory response after cold ischemia/reperfusion. Transplantation. 2007;84(10):1279-1287.

25. Kaczorowski DJ, et al. Mechanisms of Toll-like receptor 4 (TLR4)-mediated inflammation after cold ischemia/reperfusion in the heart. Transplantation. 2009;87(10):1455-1463.

26. Ding HS, et al. High mobility group [corrected] box 1 mediates neutrophil recruitment in myocardial ischemia-reperfusion injury through toll like receptor 4-related pathway. Gene. 2012;509(1):149-153.

27. Zhu H, Li J, Wang S, Liu K, Wang L, Huang L. Hmgb1-TLR4-IL-23-IL-17A axis promote ischemia-reperfusion injury in a cardiac transplantation model. Transplantation. 2013;95(12):1448-1454.

28. Tsung A, et al. Hepatic ischemia/reperfusion injury involves functional TLR4 signaling in nonparenchymal cells. J Immunol. 2005;175(11):7661-7668.

29. Zhai Y, et al. Type I, but not type II, interferon is critical in liver injury induced after ischemia and reperfusion. Hepatology. 2008;47(1):199-206.

30. Zhai Y, et al. Cutting edge: TLR4 activation medi- ates liver ischemia/reperfusion inflammatory response via IFN regulatory factor 3-dependent MyD88-independent pathway. J Immunol. 2004;173(12):7115-7119.

31. Zhou H, Andonegui G, Wong CH, Kubes P. Role of endothelial TLR4 for neutrophil recruitment into central nervous system microvessels in systemic inflammation. J Immunol. 2009;183(8):5244-5250.

32. Andonegui G, Goyert SM, Kubes P. Lipopolysaccharide-induced leukocyte-endothelial cell interactions: a role for CD14 versus toll-like receptor 4 within microvessels. Jimmunol. 2002;169(4):2111-2119.

33. McAvoy EF, McDonald B, Parsons SA, Wong $\mathrm{CH}$, Landmann R, Kubes P. The role of CD14 in neutrophil recruitment within the liver microcirculation during endotoxemia. J Immunol. 2011;186(4):2592-2601.

34. Andonegui G, et al. Endothelium-derived Tolllike receptor-4 is the key molecule in LPS-induced neutrophil sequestration into lungs. J Clin Invest. 2003;111(7):1011-1020.

35. Pasparakis M, Vandenabeele P. Necroptosis and its role in inflammation. Nature. 2015;517(7534):311-320.

36. Gao M, Monian P, Quadri N, Ramasamy R, Jiang $\mathrm{X}$. Glutaminolysis and transferrin regulate ferroptosis. Mol Cell. 2015;59(2):298-308.

37. Dixon SJ, et al. Ferroptosis: an iron-dependent form of nonapoptotic cell death. Cell. 2012;149(5):1060-1072.

38. Linkermann A, et al. Synchronized renal tubular cell death involves ferroptosis. Proc Natl Acad Sci USA. 2014;111(47):16836-16841.

39. Martin-Sanchez D, et al. Ferroptosis, but not necroptosis, is important in nephrotoxic folic acid-induced AKI. JAm Soc Nephrol. 2017;28(1):218-229.

40. Li Q, et al. Inhibition of neuronal ferroptosis protects hemorrhagic brain. JCI Insight. 2017;2(7):e90777.

41. Jiang L, et al. Ferroptosis as a p53-mediated activity during tumour suppression. Nature. 2015;520(7545):57-62.

42. Dixon SJ, Stockwell BR. The role of iron and reactive oxygen species in cell death. Nat Chem Biol. 2014;10(1):9-17.

43. Doll S, et al. ACSL4 dictates ferroptosis sensitivity by shaping cellular lipid composition. Nat Chem Biol. 2017;13(1):91-98.
44. Yang WS, et al. Regulation of ferroptotic cancer cell death by GPX4. Cell. 2014;156(1-2):317-331.

45. Dhalla NS, Elmoselhi AB, Hata T, Makino N. Status of myocardial antioxidants in ischemia-reperfusion injury. Cardiovasc Res. 2000;47(3):446-456.

46. Lejay A, et al. Ischemia reperfusion injury, ischemic conditioning and diabetes mellitus. JMol Cell Cardiol. 2016;91:11-22.

47. Liu B, Zhao C, Li H, Chen X, Ding Y, Xu S. Puerarin protects against heart failure induced by pressure overload through mitigation of ferroptosis. Biochem Biophys Res Commun. 2018;497(1):233-240.

48. Koudstaal S, et al. Necrostatin-1 alleviates reperfusion injury following acute myocardial infarction in pigs. Eur J Clin Invest. 2015;45(2):150-159.

49. Pavlosky A, et al. RIPK3-mediated necroptosis regulates cardiac allograft rejection. Am J Transplant. 2014;14(8):1778-1790.

50. Martin-Sanchez D, et al. TWEAK and RIPK1 mediate a second wave of cell death during AKI. Proc Natl Acad Sci USA. 2018;115(16):4182-4187.

51. El-Sawy T, Belperio JA, Strieter RM, Remick DG, Fairchild RL. Inhibition of polymorphonuclear leukocyte-mediated graft damage synergizes with short-term costimulatory blockade to prevent cardiac allograft rejection. Circulation. 2005;112(3):320-331.

52. Su CA, Iida S, Abe T, Fairchild RL. Endogenous memory CD8 $\mathrm{T}$ cells directly mediate cardiac allograft rejection. Am J Transplant. 2014;14(3):568-579.

53. Ardehali A, et al. Ex-vivo perfusion of donor hearts for human heart transplantation (PROCEED II): a prospective, open-label, multicentre, randomised non-inferiority trial. Lancet. 2015;385(9987):2577-2584.

54. Kreisel D, et al. Non-hematopoietic allograft cells directly activate $\mathrm{CD} 8+\mathrm{T}$ cells and trigger acute rejection: an alternative mechanism of allorecognition. Nat Med. 2002;8(3):233-239.

55. Pinto AR, et al. Revisiting cardiac cellular composition. Circ Res. 2016;118(3):400-409.

56. Folch J, Lees M, Sloane Stanley GH. A simple method for the isolation and purification of total lipides from animal tissues. J Biol Chem. 1957;226(1):497-509

57. Liu X, et al. Oxidized fatty acid analysis by charge-switch derivatization, selected reaction monitoring, and accurate mass quantitation. Anal Biochem. 2013;442(1):40-50. 